\title{
Determining atmospheric electric fields through radio emission from air showers
}

\author{
Gia Trinh ${ }^{1}$, Olaf Scholten ${ }^{1,2, \star}$, Ute Ebert ${ }^{3,4}$, Hidde Leijnse ${ }^{5}$, Casper Rutjes ${ }^{3}$, and LOFAR, Cosmic \\ ray KSP
}

${ }^{1}$ KVI-Center for Advanced Radiation Technology, University Groningen, P.O. Box 72, 9700 AB Groningen, The Netherlands

${ }^{2}$ Interuniversity Institute for High-Energy, Vrije Universiteit Brussel, Pleinlaan 2, 1050 Brussels, Belgium

${ }^{3}$ Center for Mathematics and Computer Science (CWI), PO Box 94079, 1090 GB Amsterdam, The Netherlands

${ }^{4}$ Department of Applied Physics, Eindhoven University of Technology (TU/e), PO Box 513, 5600 MB Eindhoven, The Netherlands

${ }^{5}$ Dutch Meteorological Services, KNMI, Utrechtseweg 297, 3731 GA De Bilt

\begin{abstract}
During thunderstorm conditions the radio footprint (intensity as well as polarization) of cosmic ray showers deviates strongly from those measured during fair weather. We have been able to interpret this pattern in terms of atmospheric electric fields. We see that even when there is no thunderstorm activity within $100 \mathrm{~km}$ distance within an hour of the event, the radio footprint may be strongly affected.
\end{abstract}

In recent years major advances have been made in understanding radio emission from extensive air-showers, resulting in microscopic models such as CoREAS [1] and ZHAireS [2] and macroscopic models such as MGMR [3, 4] and EVA [5]. The data agree with the predictions of these models in - for example - total radiated energy [6] and the circular polarization [7]. The models can in turn be used to extract from the measured footprint the $X_{\max }$ of the shower [8,9] which in turn is used to determine the composition of cosmic rays. To our initial surprise we also observed at LOFAR radio footprints that were completely inconsistent with model expectations [10]. These were found to be correlated with thunderstorm activity which is a clear indication that this is due to the effects of atmospheric electric fields. The supplementary material contains a somewhat extended version of this contribution.

Since the effects of these atmospheric electric fields is large it should be possible to use the footprint to extract some of the main properties of these field. This possibility was explored in [10-12].

In total we have gathered 11 of these "thunderstorm" events till August 2014 that obey certain quality conditions [13] such that they can be analyzed to extract the atmospheric fields. The results for three of these events are shown that have been measured within a time span of half an hour. Since this is rather short one can investigate to use these for a tomographic study of electric fields in clouds [13].

In these proceedings we limit ourselves to a qualitative discussion of the three events measured on December 14, 2011. A full discussion of all analyzed events is given in Ref. [13].

^e-mail: Scholten@KVI.nl 

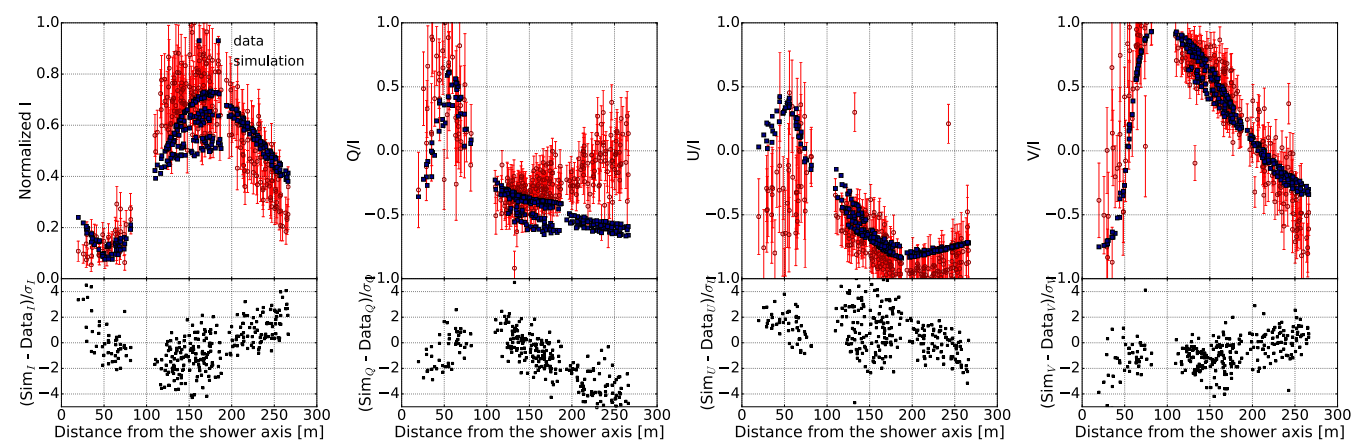

Figure 1. The Stokes parameters for event \#1 (red points) are compared to the results of a CoREAS calculation (blue points) where the parameters of the electric field have been determined from a fit using MGMR3D.

The complete footprint of the first thunderstorm event is shown in Fig. 1 where it is compared to the results for an electric field configuration. The structure of the Stokes parameters is greatly different from that for fair-weather showers; The intensity shows a ring-like structure which is an indication of fields that point in opposite directions; At large distances $Q / I$ tends to zero while $U / I$ tends to -1 implying a linear polarization at $45^{\circ}$ to the $\mathbf{v} \times \mathbf{B}$ direction; Near the core $V / I \simeq-1$ rapidly changing to $V / I \simeq+1$ at $100 \mathrm{~m}$ which implies fields that are at an angle of $90^{\circ}$. With such a complicated structure it is impressive to see that the main features of the data are reproduced this well. It is worthwhile to note that the footprints for events \#2 and \#3 differ considerably, event \#2 has a clear ring structure in intensity but polarization parameters that are similar to that of a fair-weather event while the intensity for \#3 is peaked near the shower axis, shows little circular polarization and has $U / I \simeq-1$.

The fit is performed for three different values, $X_{\max }=526,634$, and $743 \mathrm{~g} / \mathrm{cm}^{2}$. In spite of the large differences in $X_{\max }$ the results agree rather well indicating that the fit procedure is stable.

Remarkable is that during the day when the three events were detected there was no lightning activity detected within $100 \mathrm{~km}$ from the LOFAR core. It appears that at the time of the events there was a large cloud overhead. Radar reflection is an indication of the size and density of droplets and ice particles in the cloud. Lightning activity appears to be linked with radar reflection and lightning can be expected when radar reflection exceeds $40 \mathrm{dBZ}$ (yellow on the present scale). The clouds are thus not yet at the scale of active thunderclouds.

To determine the component of the electric field that is parallel to the shower direction $\left(\mathbf{E}_{\|}\right.$, which LOFAR is not sensitive to) we have to apply tomography. For this we need a few event within a small time span where the basic assumption is that the two events pass through the same electric field configuration.

The procedure is intrinsically more accurate if the two events tend to be almost perpendicular, for which reason we apply this to events \#1 and \#3 for which two events the footprint was determined with good accuracy. The results are displayed in Table 1 from which it is clear that tomography cannot be applied to the middle layers. Clearly too much has changed there. The error bars give the range of extracted values for event $i=1$ or $i=3$.

We have shown that the radio footprints of extensive air showers can be used to routinely extract the atmospheric electric fields that are perpendicular to the shower axis. When there are a few events in a short (何f hour) time span a tomographic method can be used to get the full structure. Probably due 


\begin{tabular}{|r||c|c||c|c|c|}
\hline Layer & $\vec{E}_{1} \cdot\left(e_{\vec{v}_{1} \times \vec{v}_{3}}\right)$ & $\vec{E}_{3} \cdot\left(e_{\vec{v}_{1} \times \vec{v}_{3}}\right)$ & $E_{\text {East }}$ & $E_{\text {North }}$ & $E_{\mathrm{Up}}$ \\
\hline top (1) & 3.0 & 22.6 & $-3.5 \pm 5.2$ & $-12.0 \pm 8.3$ & $-19.7 \pm 0.4$ \\
middle (2) & -89 & +45 & $-57 . \pm 36$. & $61 . \pm 57$ & $-3 . \pm 3$. \\
bottom (3) & 4.7 & 5.6 & $4.8 \pm .3$ & $-6.4 \pm .4$ & $-56.9 \pm .1$ \\
\hline
\end{tabular}

Table 1. Checking the consistency of the electric fields and the total field for the different layers using tomography for events \#1 and \#3. The quoted values are in $[\mathrm{kV} / \mathrm{m}]$.

to the fact that the shower currents are generally strongly enhanced during thunderstorm conditions there appears to be a sizable chance to detect multiple showers in such a short period.

We also observe that "thunderstorm conditions" do not necessarily imply that there is an active thunderstorm in the vicinity. Also for clouds with a large radar reflectivity we observe that the electric fields are strong enough to give rise to a footprint that is completely different from that under fair weather circumstances. It opens however the question as to what are the effects on the radio emission of less dense clouds that carry weaker electric fields.

\section{References}

[1] T. Huege, Phys. Rep. 620, 1 (2016), arXiv:1601.07426

[2] J. Alvarez-Mu niz, W.R. Carvalho, E. Zas, Astropart. Phys. 35, 325 (2012)

[3] O. Scholten, K. Werner, and F. Rusydi, Astropart. Phys. 29, 94 (2008), arXiv:0709.2872; Klaus Werner and Olaf Scholten, Astropart. Phys. 29, 393-411 (2008), arXiv:0712.2517

[4] O. Scholten, T. N. G. Trinh, K. D. de Vries, and B. M. Hare (2018), Phys. Rev. D 97, 023005 (2018)

[5] K. Werner, K.D. De Vries, O. Scholten, Astropart. Phys. 37, 5 (2012), arXiv:1201.4471

[6] A. Aab et al. (Pierre Auger Collaboration), Phys. Rev. Lett. 116, 241101 (2016); Phys. Rev. D 93, 122005 (2016); arXiv:1508.04267

[7] O. Scholten, et al., Phys. Rev. D 94, 103010 (2016), arXiv:1611.00758

[8] S. Buitink, et al., Nature, 531 (2016), p. 70

[9] K.D. de Vries, et al., Astropart. Phys. 34, 267 (2010), arXiv:1008.3308

[10] P. Schellart et al., Phys. Rev. Lett 114, 165001 (2015), arXiv:1504.05742

[11] G. Trinh, O. Scholten, et al., Phys. Rev. D 93, 023003 (2016), arXiv:1511.03045

[12] T.N.G.Trinh, et al., Phys. Rev. D 95, 083004 (2017), arXiv:1703.06008

[13] Gia Trinh, PhD thesis, University of Groningen 2018; Gia Trinh et al, submitted for publication in JGR

[14] T.C. Marshall, M.P. McCarthy, and W.D. Rust, J. Geoph. Res.: Atmosph. 100-D4, 7097 (1995).

[15] M. Stolzenburg, T.C. Marshall, and P.R. Krehbiel, , J. Geoph. Res.: Atmosph. 115-D19, 202 (2010)

[16] O. Scholten et al., contribution to these proceedings

[17] S. Buitink et al., Phys. Rev. D 90, 082003 (2014) 